\title{
Correction to: Seismic fragility functions for Portuguese RC precast buildings
}

\author{
R. Sousa ${ }^{1} \cdot$ N. Batalha ${ }^{2} \cdot$ V. Silva ${ }^{3,4} \cdot$ H. Rodrigues ${ }^{4}$ (D) \\ Published online: 17 June 2021 \\ (c) Springer Nature B.V. 2021
}

\section{Correction to: Bulletin of Earthquake Engineering https://doi.org/10.1007/s10518-020-01007-7}

In the original paper, Tables 5 and 6 were used to summarize the statistics of the fragility curves derived for the Portuguese RC Precast Buildings, namely the mean and the standard deviation. However, the values presented respects the mean and variance, not standard deviation. In this regard, the following tables replace the original ones:

The original article can be found online at https://doi.org/10.1007/s10518-020-01007-7.

\section{H. Rodrigues}

hrodrigues@ua.pt

1 CDRSP, Polytechnic of Leiria, Leiria, Portugal

2 CONSTRUCT-LESE, Faculty of Engineering (FEUP), University of Porto, Porto, Portugal

3 Faculty of Science and Technology, University Fernando Pessoa, Porto, Portugal

4 RISCO, Civil Engineering Department, University of Aveiro, Aveiro, Portugal 
Table 5 Summary of the statistics associated with the fragility functions in terms of averaged spectral accelerations in $\mathrm{m} / \mathrm{s}^{2}$

Table 6 Summary of the statistics associated with the fragility functions in terms of spectral accelerations at the elastic period in $\mathrm{m} / \mathrm{s}^{2}$

\begin{tabular}{|c|c|c|c|c|c|c|}
\hline \multirow[t]{2}{*}{ Limit state } & \multicolumn{2}{|c|}{ Pre-code } & \multicolumn{2}{|c|}{$\begin{array}{l}\text { Moderate- } \\
\text { code }\end{array}$} & \multicolumn{2}{|c|}{ Post-code } \\
\hline & Mean & SD & Mean & SD & Mean & SD \\
\hline \multicolumn{7}{|l|}{ Structural } \\
\hline Damage limitation & 0.50 & 0.37 & 0.97 & 1.37 & 1.69 & 2.48 \\
\hline Collapse prevention & 1.33 & 0.87 & 2.92 & 4.07 & 7.35 & 13.78 \\
\hline \multicolumn{7}{|l|}{ Non-structural } \\
\hline Damage limitation & 0.35 & 0.14 & 0.35 & 0.14 & 0.35 & 0.14 \\
\hline Collapse prevention & 1.50 & 0.77 & 1.44 & 0.68 & 1.40 & 0.63 \\
\hline
\end{tabular}

\begin{tabular}{|c|c|c|c|c|c|c|}
\hline \multirow[t]{2}{*}{ Limit state } & \multicolumn{2}{|c|}{ Pre-code } & \multicolumn{2}{|c|}{$\begin{array}{l}\text { Moderate- } \\
\text { code }\end{array}$} & \multicolumn{2}{|c|}{ Post-code } \\
\hline & Mean & SD & Mean & SD & Mean & SD \\
\hline \multicolumn{7}{|l|}{ Structural } \\
\hline Damage limitation & 0.50 & 0.39 & 0.98 & 1.44 & 1.76 & 2.76 \\
\hline Collapse prevention & 1.34 & 0.92 & 3.17 & 4.80 & 9.03 & 19.63 \\
\hline \multicolumn{7}{|l|}{ Non-structural } \\
\hline Damage limitation & 0.35 & 0.14 & 0.35 & 0.14 & 0.35 & 0.14 \\
\hline Collapse prevention & 1.52 & 0.83 & 1.46 & 0.73 & 1.41 & 0.66 \\
\hline
\end{tabular}

Publisher's Note Springer Nature remains neutral with regard to jurisdictional claims in published maps and institutional affiliations. 